\title{
Fatores associados à avaliação da Atenção Primária à Saúde na perspectiva do usuário: resultados do inquérito telefônico Vigitel, 2015
}

\author{
Factors associated with the evaluation of Primary Health Care from \\ the user's perspective: results of the telephone survey Vigitel, 2015
}

Rosângela Durso Perillo (https://orcid.org/0000-0003-4791-2317) ${ }^{1}$

Kátia Crestine Poças (https://orcid.org/0000-0002-1254-8001)2

Regina Tomie Ivata Bernal (https://orcid.org/0000-0002-7917-3857) ${ }^{1}$

Elisabeth Carmen Duarte (https://orcid.org/0000-0001-9148-5063) ${ }^{2}$

Deborah Carvalho Malta (https://orcid.org/0000-0002-8214-5734) ${ }^{1}$

${ }^{1}$ Departamento de Enfermagem Materno Infantil e Saúde Pública, Escola de Enfermagem, Universidade Federal de Minas Gerais. Av. Prof. Alfredo Balena 190/438, Santa Efigênia. 30130-100 Belo Horizonte MG Brasil. rosangeladurso.perillo@ gmail.com

${ }^{2}$ Faculdade de Medicina, Universidade de Brasília. Brasília DF Brasil.

\begin{abstract}
This paper aims to evaluate the performance of PHC from the perspective of users and its association with sociodemographic characteristics, self-reported health conditions, and behavioral risk factors for Chronic Noncommunicable Diseases. This is a population-based cross-sectional stu$d y$ with data from the 2015 VIGITEL Telephone Survey. The Primary Care Assessment Tool short version was adopted. The study population covers adults over 18 years of age who used PHC services in Belo Horizonte in the last 12 months $(n=872)$. The multiple logistic regression model was performed to estimate the odds ratio. We observed that adults without a health insurance plan are 3.21 (95\% CI 2.08-4.96) more likely than those with a health insurance plan to evaluate PHC with a high score ( $\geq 6.6)$, and adults with low schooling (95\% CI 1.48-5.32), people with diabetes (95\% CI 1.05-3.24), obese (95\% CI 1.20-3.24), and older adults (95\% CI 1.00-1.41) were 2.81, 1.84, 1.97, and 1.19 more likely to report a high score for PHC quality than the others, respectively. The use of the PCATool short version in a telephone survey showed a new possibility for PHC performance assessment and can become useful in managing health services.
\end{abstract}

Key words Primary Health Care, Health services assessment, Health surveillance by telephone survey
Resumo O objetivo deste artigo é avaliar o desempenho da APS sob a perspectiva dos usuários e sua associação com as características sociodemográficas, condições de saúde autorreferidas e fatores de risco comportamentais para Doenças Crônicas Não Transmissiveis. Estudo transversal de base populacional, com dados do Inquérito Telefônico Vigitel 2015. Utilizou-se o "Primary Care Assessment Tool" versão reduzida. A população do estudo são adultos maiores de 18 anos que utilizaram os serviços da APS em Belo Horizonte nos últimos 12 meses $(n=872)$. Realizado o modelo de regressão logística múltipla para estimar o odds ratio. Observa-se que adultos sem plano de saúde têm 3,21 (IC ${ }_{950}$ 2,08-4,96) mais chances do que com plano de saúde de avaliarem a APS com alto escore $(\geq 6,6)$, bem como adultos com baixa escolaridade tem 2,81 (IC $\left.{ }_{95 \%} 1,48-5,32\right)$ mais chances, os diabéticos têm 1,84 (IC $\left.C_{95 \%} 1,05-3,24\right)$ mais chances, os obesos tem 1,97 (IC I5\% $^{1,20-3,24)}$ mais chances e os idosos tem 1,19 (IC $\left.{ }_{95 \%}, 1,00-1,41\right)$ chances de reportar alto escore para a qualidade da APS do que os demais. A utilização do PCATool na versão reduzida em inquérito telefônico, mostrou-se nova possibilidade de avaliação do desempenho da APS e pode se tornar útil na gestão dos serviços de saúde.

Palavras-chave Atenção Primária à Saúde, Avaliação de serviços de saúde, Sistema de Vigilância por Inquérito Telefônico 


\section{Introdução}

A Atenção Primária à Saúde (APS) é considerada o eixo norteador da Rede de Atenção à Saúde (RAS) no Sistema Único de Saúde (SUS). É responsável por garantir o acesso universal e igualitário às ações e aos serviços de saúde disponíveis e pela diminuição das internações por condições sensíveis à atenção primária. Uma APS forte e resolutiva contribui para a redução dos custos do sistema de saúde e para a manutenção dos princípios do SUS2.

Segundo Starfield e $\mathrm{Shi}^{3}$, a APS deve ser considerada a porta de entrada do sistema de saúde, além de oferecer acesso aos serviços de prevenção, cura e reabilitação. Deve ainda racionalizar todos os recursos disponíveis para promoção e manutenção da saúde. Deve também integrar os pontos de atenção do sistema de saúde de forma a garantir, em tempo oportuno, a realização do cuidado adequado às necessidades do usuário ${ }^{3}$.

Uma APS fortalecida e bem estruturada deve apresentar quatro elementos estruturais ou chamados de essenciais: a) primeiro contato; b) longitudinalidade; c) integralidade; e d) coordenação. Além desses, deve apresentar dois elementos chamados de derivados: centralização na família e orientação comunitária ${ }^{4}$. Assim, um dos marcos de referência para a avaliação de serviços da APS são a avaliação desses atributos.

Mesmo com os avanços ocorridos nas últimas décadas no âmbito da saúde com a consolidação do SUS e a implantação da Estratégia da Saúde da Família (ESF) ${ }^{5}$, é importante garantir assistência de qualidade que atenda às necessidades dos usuários. Para que se possa qualificar os serviços é importante realizar processos avaliativos com abordagens que mostrem as perspectivas dos diversos atores envolvidos na atenção à saúde, como os gestores, os profissionais e os usuários. A avaliação contribui também na identificação dos entraves e das fragilidades dos serviços de APS ${ }^{6,7}$.

A avaliação dos serviços de saúde deve ser entendida como um instrumento de gestão presente em todas as ações de saúde. Pode direcionar e/ou redirecionar as políticas e os programas de saúde promovendo e qualificando a atenção à saúde e fortalecendo os princípios do SUS5. Contribui, ainda, no controle social quando os resultados são compartilhados com a população, favorecendo a participação no processo de tomada de decisão dos gestores ${ }^{6-8}$.

Após 30 anos da origem do SUS, foi criada a Secretaria de Atenção Primária à Saúde (SAPS) enfatizando assim a relevância da APS enquanto prioridade do SUS. Entre os objetivos da SAPS estão o fortalecimento dos atributos essenciais da APS, bem como dos atributos derivados, formação e provimento profissional, estratégias de suporte assistencial, desenvolvimento de tecnologias de informação e cuidado, entre outros?

Considerando a avaliação da APS, foram desenvolvidos alguns instrumentos utilizados em diversos países. Fracolli et al. ${ }^{10}$, em uma revisão e metassíntese realizada no período de 1979 e 2013, identificou os principais instrumentos utilizados nacional e internacionalmente na avaliação da APS. Afirmou ainda que o Primary Care Assessment Tool (PCATool ${ }^{11}$ é o instrumento mais utilizado no Brasi $1^{10}$. Ribeiro e Scatena ${ }^{12}$ em outro estudo bibliográfico da produção científica entre 2007 e 2017 sobre a avaliação da APS no contexto brasileiro, também observou que o PCAToo ${ }^{11}$ foi o instrumento mais utilizado nos estudos publicados neste período. Este instrumento tem grande relevância considerando que já foi validado e utilizado em vários países e nas diversas regiões do Brasil, permitindo, assim, estabelecer comparações dos resultados obtidos nesta pesquisa com outros estudos ${ }^{12}$.

Outra questão importante se refere ao perfil dos usuários dos serviços de saúde. O estudo de Malta et al. ${ }^{13}$ confirmou a maior utilização destes serviços por pessoas com Doenças Crônicas Não Transmissíveis (DCNT), fato este que pode ser explicado pela maior demanda de consultas de rotina ou intercorrências, maior comorbidades associadas, bem como a necessidade de um monitoramento contínuo ${ }^{14,15}$.

As condições crônicas são um grave problema de saúde pública ${ }^{16} \mathrm{e}$ acarretam altos custos para o sistema de saúde. Têm ainda grande impacto na qualidade de vida da população, o que nos mostra que a APS tem papel fundamental por representar o elo do sistema de saúde responsável pelo monitoramento destes casos, que muitas vezes exigem cuidados mais complexos e coordenados entre diversos serviços.

Vários fatores de risco estão relacionados às DCNT, tais como, a alimentação inadequada, a ingestão excessiva de sal, o consumo abusivo de álcool, a inatividade física, o excesso de peso, o tabagismo e os distúrbios do metabolismo da glicose e dos lipídios ${ }^{17}$. Estes fatores de risco são alvo de intervenções contempladas nas políticas de saúde, principalmente no âmbito da APS.

Neste contexto, este estudo tem relevância considerando a escassez de trabalhos de avaliação do desempenho da APS na perspectiva do usuário, bem como estudos que apresentem análise 
relacionando a avaliação do desempenho da APS com os desfechos clínicos. Ademais, é de grande importância a avaliação da APS utilizando uma base de dados populacional nacional como o Sistema de Vigilância de Fatores de Risco e Proteção para Doenças Crônicas por Inquérito Telefônico $(\text { Vigitel })^{18}$. Foi usado pela primeira vez para este fim e apresenta-se assim, como uma possibilidade inovadora e de baixo custo.

Considerando o exposto acima, este estudo, realizado em Belo Horizonte mediante uso do PCATool, tem como objetivo avaliar o desempenho da APS sob a perspectiva dos usuários e sua associação com as características sociodemográficas, condições de saúde autorreferidas e fatores de risco comportamentais para DCNT.

\section{Métodos}

Trata-se de estudo transversal de base populacional. Foram utilizados dados da amostra de Belo Horizonte do Vigitel $2015^{18}$. Este estudo foi realizado no município de Belo Horizonte (BH), capital do estado de Minas Gerais. Em 2019, a APS da rede o SUS-BH alcançou uma a cobertura de $80,82 \%$, contando com uma estrutura de 152 Centros de Saúde, 592 equipes de Saúde da Família, 304 equipes de Saúde Bucal, 152 equipes de Saúde Mental, 82 polos de Núcleo Ampliado de Saúde da Família e Atenção Básica e 78 Academias da Cidade ${ }^{19}$.

O Vigitel 2015 entrevistou por meio de questionário estruturado, a população adulta $(\geq 18$ anos de idade) residente em domicílios com pelo menos uma linha telefônica fixa ${ }^{18}$. A entrevista telefônica inicia com a utilização de um questionário do Vigitel 2015 composto por questões que abordam as características demográficas e socioeconômicas dos indivíduos, fatores de risco comportamentais para DCNT e condições de saúde autorreferida ${ }^{18}$. Após a aplicação deste questionário, os entrevistados responderam perguntas em busca de identificar aqueles que utilizaram algum serviço de saúde nos últimos 12 meses $^{20,21}$. São elas:

- "Quando está doente ou precisando de atendimento para cuidar da própria saúde, qual serviço de saúde o(a) senhor(a) costuma procurar?" (Se público ou privado, seja APS, hospital ou serviço de urgência);

- "Nos últimos 12 meses, o(a) senhor(a) procurou atendimento em uma Unidade Básica de Saúde (UBS) (seja um posto de saúde ou centro de saúde ou unidade de saúde da família) para cuidar da própria saúde? (Sim ou não). Se sim, quantas vezes?".

Assim para este estudo foi considerado o adulto entrevistado que respondeu ter procurado algum serviço de saúde da APS pelo menos uma vez nos últimos 12 meses e que mencionaram o nome ou localização da UBS procurada no município de Belo Horizonte ${ }^{20,21}$. Esses entrevistados foram então convidados a responder o módulo avaliação Vigitel com a intenção de fazer a avaliação do desempenho dos serviços da APS do município $^{22}$.

Neste estudo utilizou-se apenas a parte do módulo avaliação Vigitel composta pelo PCATool -Adulto-Brasil versão reduzida para usuários dos serviços de APS, traduzido para o português e validado no Brasil ${ }^{23}$. Este instrumento tem 23 itens dispostos em blocos de perguntas que correspondem à avaliação de desempenho dos atributos da APS (acesso, longitudinalidade, integralidade, coordenação, orientação familiar e orientação

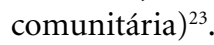

O PCATool constitui uma ferramenta de avaliação da APS desenvolvida em Baltimore, Maryland (EUA), por Starfield e colaboradores na The Johns Hopkins Populations Care Policy Center for the Underserved Populations e visa medir a presença e a extensão dos atributos da $\mathrm{APS}^{24}$. O instrumento foi criado com base no modelo de avaliação da qualidade de serviços de saúde proposto por Donabedian ${ }^{25}$, cuja avaliação apoia-se na mensuração de aspectos de estrutura, processo e resultados dos serviços de saúde. $\mathrm{O}$ PCATool ${ }^{11}$ propõe medir a presença e a extensão dos atributos da APS segundo os aspectos de estrutura e processo. Potencializado por métodos estatísticos, o PCATool possibilita a associação à efetividade das ações e serviços prestados, bem como estabelecer associações a outros desfechos clínicos ${ }^{26,27}$.

As respostas aos itens do PCATool utilizam a escala do tipo Likert onde o entrevistado especifica seu nível de concordância com o item apresentado, variando de 1 a 4 para a análise de cada atributo ( $1=$ com certeza não; $2=$ provavelmente não; $3=$ provavelmente sim; $4=$ com certeza sim), com o acréscimo da opção 9 (não sei/ não lembro $)^{11}$. Após a consolidação dos dados de cada atributo, os valores são transformados em uma escala contínua, variando entre zero e dez (Quadro 1) ${ }^{11}$.

Além do cálculo do escore por atributo avalia-se também os escores essencial, derivado e geral. Para a realização das análises estatísticas calculou-se também o que chamamos de escore 
Quadro 1. Descrição dos cálculos dos escores do PCATool.

\begin{tabular}{|c|c|c|}
\hline Escore & Cálculo & Descriçãao \\
\hline Escore geral padronizado & $\begin{array}{l}\text { (escore-escore mínimo })^{*} 10 \\
\text { escore máximo-escore mínimo }\end{array}$ & $\begin{array}{l}\text { (1) se escore geral padronizado } \geq 6,6 \\
\text { (0) se escore geral padronizado }<6,6\end{array}$ \\
\hline Escore Geral & $\frac{\mathrm{A}+\mathrm{B}+\mathrm{C}+\mathrm{D}+\mathrm{E}+\mathrm{F}+\mathrm{G}+\mathrm{H}+\mathrm{I}}{9}$ & $\begin{array}{l}\text { Soma do grau de afiliação mais } \\
\text { escore médio dos componentes que } \\
\text { pertencem aos atributos essenciais e } \\
\text { derivados, dividido pelo número total de } \\
\text { componentes }\end{array}$ \\
\hline Escore Essencial & $\frac{\mathrm{A}+\mathrm{B}+\mathrm{C}+\mathrm{D}+\mathrm{E}+\mathrm{F}+\mathrm{G}}{7}$ & $\begin{array}{l}\text { Soma do grau de afiliação mais escore } \\
\text { médio entre os componentes dos atributos } \\
\text { primeiro contato (B), longitudinalidade } \\
\text { (C), coordenação (D e E) e integralidade } \\
\text { disponível (F), dos serviços prestados (G), } \\
\text { somados ao grau de afiliação (A) }\end{array}$ \\
\hline Escore Derivado & $\frac{\mathrm{H}+\mathrm{I}}{2}$ & $\begin{array}{l}\text { Soma da média dos atributos enfoque } \\
\text { familiar }(\mathrm{H}) \text { e orientação comunitária (I) }\end{array}$ \\
\hline Escore por atributo & $\begin{array}{l}\text { Após a consolidação dos dados } \\
\text { relativos de cada atributo, os valores } \\
\text { são transformados em uma escala } \\
\text { contínua, variando entre zero (0) e } \\
\text { dez (10) dada pela expressão [escore } \\
\text { obtido - } 1 \text { (valor mínimo)] X } 10 \text { / } 4 \\
\text { (valor máximo) - } 1 \text { (valor mínimo) }\end{array}$ & $\begin{array}{l}\text { (A) afiliação } \\
\text { (B) utilização } \\
\text { (C) atendimento } \\
\text { (D) coordenação de cuidado } \\
\text { (E) coordenação de informação } \\
\text { (F) integralidade disponível } \\
\text { (G) integralidade prestado } \\
\text { (H) enfoque familiar } \\
\text { (I) orientação comunitária }\end{array}$ \\
\hline
\end{tabular}

Nota: O grau de afiliação visa a identificar o serviço ou profissional de saúde (médico/enfermeiro) que serve como referência para o cuidado, o que não é considerado um atributo da APS, porém é utilizado no cálculo dos escores essencial e geral.

geral padronizado, que representa o ponto de corte considerando o escore geral encontrado (Quadro 1) $)^{11}$.

Um escore geral $\geq 6,6$ evidencia uma forte orientação à APS, o que equivale na escala do tipo Likert o valor 3 (provavelmente sim) e consequentemente uma boa qualidade da assistência (Quadro 1) ${ }^{11}$.

Vale ressaltar que o grau de afiliação visa identificar o profissional ou o serviço que serve como referência para o entrevistado e, portanto, não é considerado atributo da APS, mas é incluído no cálculo dos escores essencial e geral ${ }^{11}$.

$\mathrm{Na}$ amostra Vigitel Belo Horizonte 2015, das 3.800 linhas telefônicas utilizadas (equivalente a 19 réplicas de 200 números de telefones cada) foram realizadas 2.125 entrevistas, nas quais, 2.006 entrevistados relataram ter procurado algum serviço de saúde quando precisaram de atendimento e, destes, 795 usuários responderam ao instrumento, o Vigitel completo e o módulo avaliação Vigitel (Figura 1).

A população do estudo foi composta por adultos usuários da APS e que aceitaram responder o módulo avaliação Vigitel. O tamanho da amostra foi definido como sendo 1.000 adultos e foi obtida pela expressão:

$$
\begin{aligned}
& \mathrm{n}=\frac{\mathrm{p} \cdot(1-p)}{(d / z)^{2}}, \text { sendo } \mathrm{p}=50 \%, z \text { igual a } 1.96 \\
& \text { e margem de erro de } 3.1
\end{aligned}
$$

A amostra obtida com o módulo avaliação Vigitel foi de 795 entrevistas e para alcançar o tamanho mínimo definido pelo cálculo amostral foi necessário acrescentar cinco réplicas com 200 números de telefones em cada, totalizando 1.000 números de telefones. Destes, entrevistaram-se mais 118 adultos, que responderam ao questionário reduzido do Vigitel e o módulo avaliação Vigitel, totalizando, assim, 913 entrevistas. Excluíram-se 41 entrevistas em virtude da impossibilidade de localização do endereço da Unidade Básica de Saúde (UBS) a qual o entrevistado disse ter utilizado (Figura 1).

Desse modo, a população deste estudo são adultos maiores de 18 anos de idade residentes em domicílios servidos por, pelo menos, uma linha telefônica fixa de Belo Horizonte, que utilizaram os serviços da APS nesse município nos últimos 12 meses anteriores à entrevista, com 


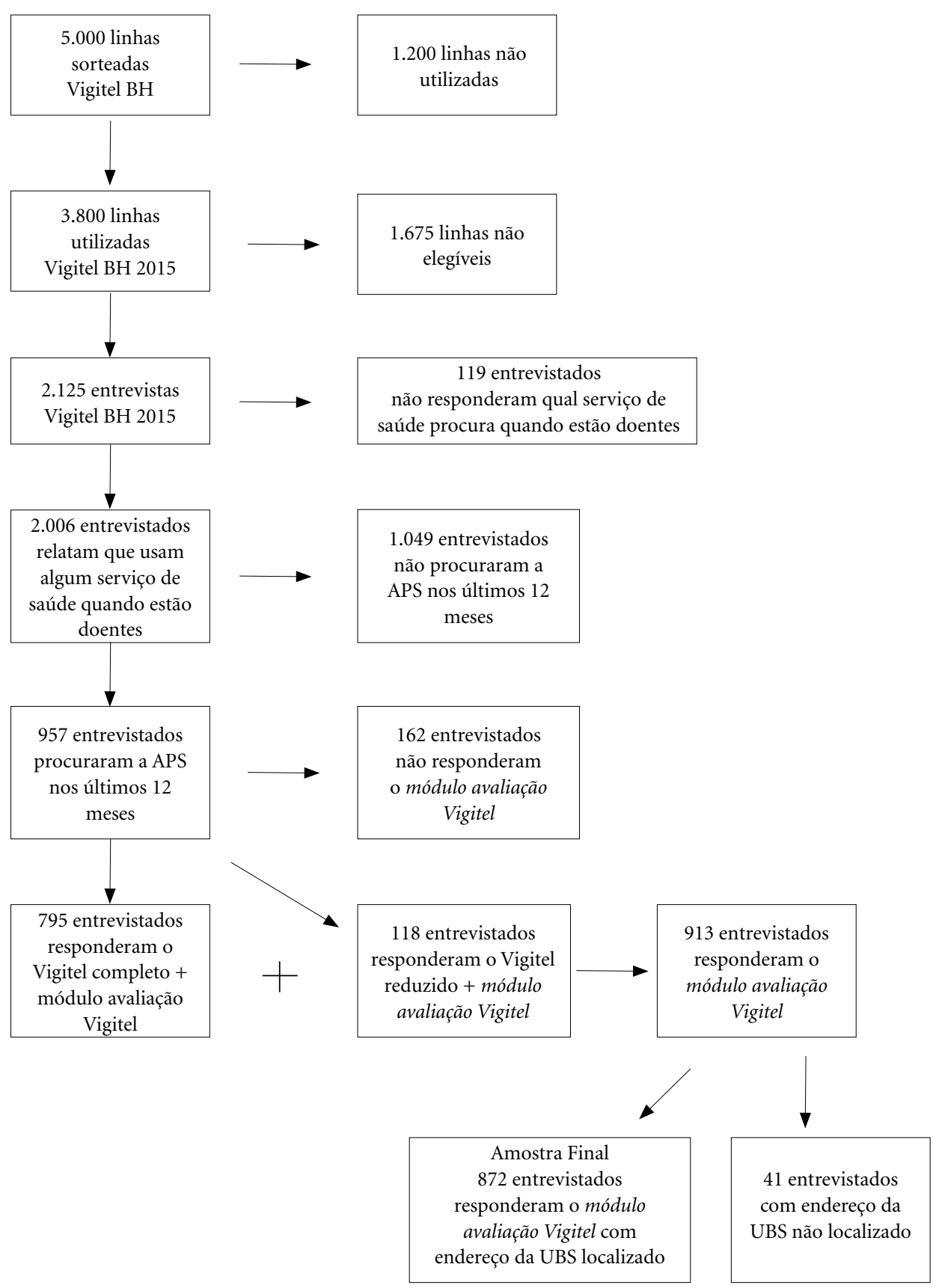

Figura 1. Fluxo amostral do estudo da análise do desempenho dos serviços de APS na perspectiva do usuário. Belo Horizonte, Brasil. Vigitel, 2015.

endereço da UBS localizado e que aceitaram responder o módulo avaliação Vigitel. $(\mathrm{n}=872)$ (Figura 1).

Visando reduzir viés de seleção da amostra Vigitel Belo Horizonte 2015 que entrevista adultos com linha telefônica fixa, aplicou-se pro- cedimentos pós-estratificação calculados pelo método rake para expansão da amostra para a população total. O detalhamento sobre o delineamento amostral da pesquisa Vigitel e do tratamento pós-estratificação foram descritos em outras publicações ${ }^{18,28}$. 
Novos pesos de pós-estratificação foram calculados para ajustar a distribuição de usuários de APS segundo idade, sexo e escolaridade. Esses pesos foram calculados no programa Data Analysis and Statistical Softwares (STATA) versão 14.0 usando o pacote SURVWGT mediante uso do método rake e a estimativa da população usuária da APS obtida no módulo avaliação Vigitel como população de referência ${ }^{29,30}$.

Para caracterizar os usuários do serviço da APS em BH realizou-se a análise descritiva das variáveis por meio das frequências absolutas e relativas. Em um segundo momento utilizou-se o teste 2 de Person para identificar associações, com nível de significância de 5\%.

A variável desfecho deste estudo (extraídas do módulo avaliação Vigitel) foi o escore geral padronizado (se $\geq 6,6$ ou $<6,6$ ). As variáveis explicativas (extraídas do questionário Vigitel) podem ser descritas em três grupos. O primeiro deles são as características sociodemográficas onde foram analisadas as variáveis sexo (masculino; feminino), faixa etária (em anos: 18-29; 30-39; 40-59; 60 ou mais), escolaridade (anos de estudo: 0 a 8 ; 9 a $11 ; 12$ ou mais), raça/cor (branca; preta, amarela; parda; indígena), situação conjugal (com ou sem companheiro) e ter plano de saúde (sim ou não). O segundo grupo contempla os fatores de risco comportamentais para as DCNT selecionados no qual selecionou-se as variáveis uso de tabaco (sim ou não), isto é, os entrevistados que se declararam fumantes independentemente do número de cigarros, da frequência e da duração do hábito de fumar e o uso abusivo de álcool (sim ou não). Neste último fator de risco foi considerado aquele entrevistado que relatou consumir cinco ou mais doses (homem) ou quatro ou mais doses (mulher) em uma única ocasião, pelo menos uma vez nos últimos 30 dias. E o terceiro grupo analisa as condições de saúde autorreferida, como o relato de diagnóstico médico prévio de diabetes, hipertensão arterial; obesidade (IMC $\geq 30 \mathrm{~kg} / \mathrm{m} 2$, calculados a partir de peso e altura autorreferidos).

Para a análise da associação de interesse entre o escore da APS e as variáveis explicativas foi utilizado o modelo de regressão logística múltipla para estimar o Odds Ratio (OR) bruto e ajustado por sexo e idade e os respectivos intervalos de confiança (IC) de 95\%.

Todas as análises foram realizadas utilizando o módulo Survey disponível no Data Analysis and Statistical Softwares (STATA) versão 14.0 para que fosse possível incorporar a ponderação dos dados do Vigitel.
A realização do Vigitel foi aprovada pela Comissão Nacional de Ética em Pesquisa (CONEP), e a pesquisa foi aprovada pelo Comitê de Ética em Pesquisa da Faculdade de Medicina da UnB, (CEP/FM/UnB). Dada a natureza das entrevistas, substituiu-se o consentimento livre e esclarecido pelo consentimento verbal obtido por ocasião dos contatos telefônicos com os entrevistados.

\section{Resultados}

Do total de 2.125 entrevistados, em 2015, em Belo Horizonte, quando indagados sobre a utilização dos serviços da APS nos últimos 12 meses, 872 usuários responderam positivamente $(41,04 \%)$.

Dos 872 entrevistados que avaliaram a APS a maioria são mulheres (69,38\%), idosos (44,04\%), que se declaram pardos $(44,06 \%)$, com plano de saúde $(51,61 \%)$, com escolaridade menor que 11 anos de estudo $(69,38 \%)$, sendo que destes $38,88 \%$ tem menos de 8 anos de estudo. Em relação à situação de saúde autorreferida e fatores de risco para as DCNT, 41,74\% são hipertensos, 13,19\% diabéticos, 20,51\% obesos, $8,03 \%$ fumantes e 10,44\% relataram uso abusivo de álcool.

$\mathrm{Na}$ avaliação da presença e extensão dos atributos da APS, segundo escore geral obtido, $19,61 \%(\mathrm{n}=171)$ dos usuários avaliaram com escore $\geq 6,6$, enquanto que com $80,39 \%(n=701)$ o escore dado foi $<6,6$ (Tabela 1 ).

A Tabela 1 descreve o perfil dos usuários dos serviços da APS, segundo escore geral de avaliação. Observa-se que, dentre os que melhor avaliaram a APS (escore $\geq 6,6$ ), a maioria dos usuários tem baixa escolaridade, isto é $\leq 8$ anos de estudo $\left(27,08 \% ; \mathrm{IC}_{95 \%} 21,72-33,20\right)$ e não tem plano de saúde $\left(28,85 \% ; \mathrm{IC}_{95 \%} 23,96-34,28\right)$. Em relação aos que avaliaram negativamente estão os adultos com maior escolaridade, ou seja 12 ou mais anos de estudo $\left(89,29 \%\right.$; $\left.\mathrm{IC}_{95 \%} 82,74-93,55\right)$, e os que têm plano de saúde $\left(88,93 \%\right.$ IC $\left._{95 \%} 84,94-91,96\right)$.

A Tabela 2 apresenta a avaliação dos usuários dos serviços de APS, segundo fatores de risco comportamentais para as DCNT e as condições de saúde autorreferidas. Observa-se que, dentre os que melhor avaliaram a APS (escore $\geq 6,6$ ) estão os usuários hipertensos $\left(26,59 \%\right.$; $\mathrm{IC}_{95 \%} 21,33$ $32,60)$, diabéticos $\left(32,94 \%\right.$ IC $\left._{95 \%} 23,11-44,52\right)$ e obesos $\left(31,23 \%\right.$ IC $\left._{95 \%} 23,16-40,64\right)$.

A Tabela 3 apresenta o resultado encontrado na aplicação do modelo de regressão logística múltipla. No modelo bruto pode-se observar que os usuários sem plano de saúde têm 3,26 mais chances $\left(\mathrm{IC}_{95 \%} 2,11-5,03\right)$ de reportar alto escore 
Tabela 1. Caracterização dos usuários adultos dos serviços de Atenção Primária à Saúde nos últimos 12 meses, segundo características sociodemográficas e escores de avaliação. Vigitel, Belo Horizonte, 2015. ( $\mathrm{n}=872$ )

\begin{tabular}{|c|c|c|c|c|c|c|c|}
\hline \multirow[t]{2}{*}{ Variáveis } & \multicolumn{3}{|c|}{$\begin{array}{c}\text { Escore } \geq 6,6^{a} \\
n=171\end{array}$} & \multicolumn{3}{|c|}{$\begin{array}{c}\text { Escore }<6,6^{b} \\
n=701\end{array}$} & \multirow[t]{2}{*}{ Valor de $\mathrm{p}^{\mathrm{d}}$} \\
\hline & $\%^{c}$ & & & $\%^{\mathrm{c}}$ & & $\%^{\mathrm{d}}$ & \\
\hline Masculino & 23,27 & 17,77 & 29,85 & 76,73 & 70,15 & 82,23 & \\
\hline Feminino & 18,96 & 15,44 & 23,07 & 81,04 & 76,93 & 84,56 & \\
\hline Faixa etária (anos) & & & & & & & 0,181 \\
\hline 18 a 29 & 14,26 & 8,30 & 23,42 & 85,74 & 76,58 & 91,70 & \\
\hline 30 a 39 & 18,08 & 11,27 & 27,73 & 81,92 & 72,27 & 88,73 & \\
\hline 40 a 59 & 22,00 & 16,75 & 28,34 & 78,00 & 71,66 & 83,25 & \\
\hline 60 ou mais & 24,86 & 19,80 & 30,70 & 75,14 & 69,30 & 80,20 & \\
\hline Escolaridade (anos de estudo) & & & & & & & 0,001 \\
\hline 12 ou mais & 10,71 & 6,45 & 17,26 & 89,29 & 82,74 & 93,55 & \\
\hline 9 a 11 & 18,83 & 14,27 & 24,42 & 81,17 & 75,58 & 85,73 & \\
\hline 0 A 8 & 27,08 & 21,72 & 33,20 & 72,92 & 66,80 & 78,28 & \\
\hline Raça/cor* & & & & & & & 0,349 \\
\hline Branca & 18,89 & 14,17 & 24,73 & 81,11 & 75,27 & 85,83 & \\
\hline Preta & 18,77 & 11,96 & 28,21 & 81,23 & 71,79 & 88,04 & \\
\hline Amarela & 11,04 & 3,16 & 32,05 & 88,96 & 67,95 & 96,84 & \\
\hline Parda & 23,95 & 18,94 & 29,81 & 76,05 & 70,19 & 81,06 & \\
\hline Indígena & 8,90 & 1,17 & 44,67 & 91,10 & 55,33 & 98,83 & \\
\hline Situação Conjugal & & & & & & & 0,199 \\
\hline Com companheiro(a) & 22,47 & 18,25 & 27,34 & 77,53 & 72,66 & 81,75 & \\
\hline Sem companheiro(a) & 18,18 & 14,01 & 23,27 & 81,82 & 76,73 & 85,99 & \\
\hline Plano de saúde & & & & & & & 0,000 \\
\hline Sim & 11,07 & 8,04 & 15,06 & 88,93 & 84,94 & 91,96 & \\
\hline Não & 28,85 & 23,96 & 34,28 & 71,15 & 65,72 & 76,04 & \\
\hline
\end{tabular}

(a) $n=171$ (b) $n=701$ (c) Percentual ponderado para ajustar a distribuição sociodemográfica da amostra Vigitel à distribuição da população adulta do BH (d) Valor de $\mathrm{p}$ do teste do $\chi^{2}$ de Pearson (escore $\geq 6,6$ e escore $<6,6$ ). (d) IC $95 \%$ : intervalo de confiança de $95 \%$.

Nota: Categorização dos entrevistados do Vigitel 2015 que responderam o módulo de avaliação da APS dos itens relacionados ao PCATool-Brasil-Adulto versão reduzida e com endereço localizado.

$(\geq 6,6)$ para a qualidade da APS do que os demais, os usuários com baixa escolaridade, ou seja, com menos de 8 anos de estudo, tem 3,10 mais chances $\left(\mathrm{IC}_{95 \%} 1,66-5,79\right)$, bem como os usuários com 9 a 11 anos de estudo tem 1,93 mais chances $\left(\mathrm{IC}_{95 \%} 1,01-1,41\right)$, os obesos têm 2,04 mais chances ( $\left.\mathrm{IC}_{95 \%} 1,28-3,57\right)$, os diabéticos têm 2,08 mais chances $\left(\mathrm{IC}_{95 \%} 1,14-3,57\right)$, os usuários hipertensos tem 1,70 mais chances $\left(\mathrm{IC}_{95 \%} 1,14-2,53\right)$ e os idosos (com 60 anos e mais) têm $1,19\left(\mathrm{IC}_{95 \%} 1,00\right.$ $1,41)$ mais chances de reportar alto escore do que os usuários de outras faixas etárias.

No modelo ajustado pelas variáveis confundidoras (idade e sexo), observa-se que usuários sem plano de saúde têm 3,21 mais chances $\left(\mathrm{IC}_{95 \%}\right.$ $2,08-4,96)$ de reportar alto escore $(\geq 6,6)$ para a qualidade da APS, do que adultos com plano de saúde, enquanto que usuários com baixa escolaridade ( 0 a 8 anos de estudo) têm 2,81 mais chances $\left(\mathrm{IC}_{95 \%}\right.$ 1,48-5,32) de reportar alto escore, bem como os usuários com 9 a 11 anos de estudo têm 2,00 mais chances $\left(\mathrm{IC}_{95 \%} 1,03-3,89\right)$ e os idosos têm 1,19 ( IC $\left._{95 \%} 1,00-1,41\right)$ mais chances de reportar alto escore do que os usuários de outras faixas etárias (Tabela 3 ).

Considerando as condições de saúde autorreferidas, os diabéticos têm 1,84 mais chances $\left(\mathrm{IC}_{95 \%} 1,05-3,24\right)$ de reportar alto escore, bem como os obesos têm 1,97 mais chances ( IC $_{95 \%}$ 1,20-3,24).

Em relação a faixa etária, idosos (acima de 60 anos) têm 1,19 chances $\left(\mathrm{IC}_{95 \%} 1,00-1,41\right)$ de reportar alto escore para a qualidade da APS, do que adultos em outras faixas etárias.

O desfecho "hipertensão arterial" perdeu significância estatística $(p=0,095)$ e não conferiu diferença após aplicação do modelo ajustado (Tabela 3). 
Tabela 2. Caracterização dos usuários adultos dos serviços de Atenção Primária à Saúde nos últimos 12 meses, segundo fatores de risco comportamentais para as Doenças Crônicas Não Transmissíveis, condições de saúde autorreferida e escores de avaliação, Vigitel, Belo Horizonte, 2015.

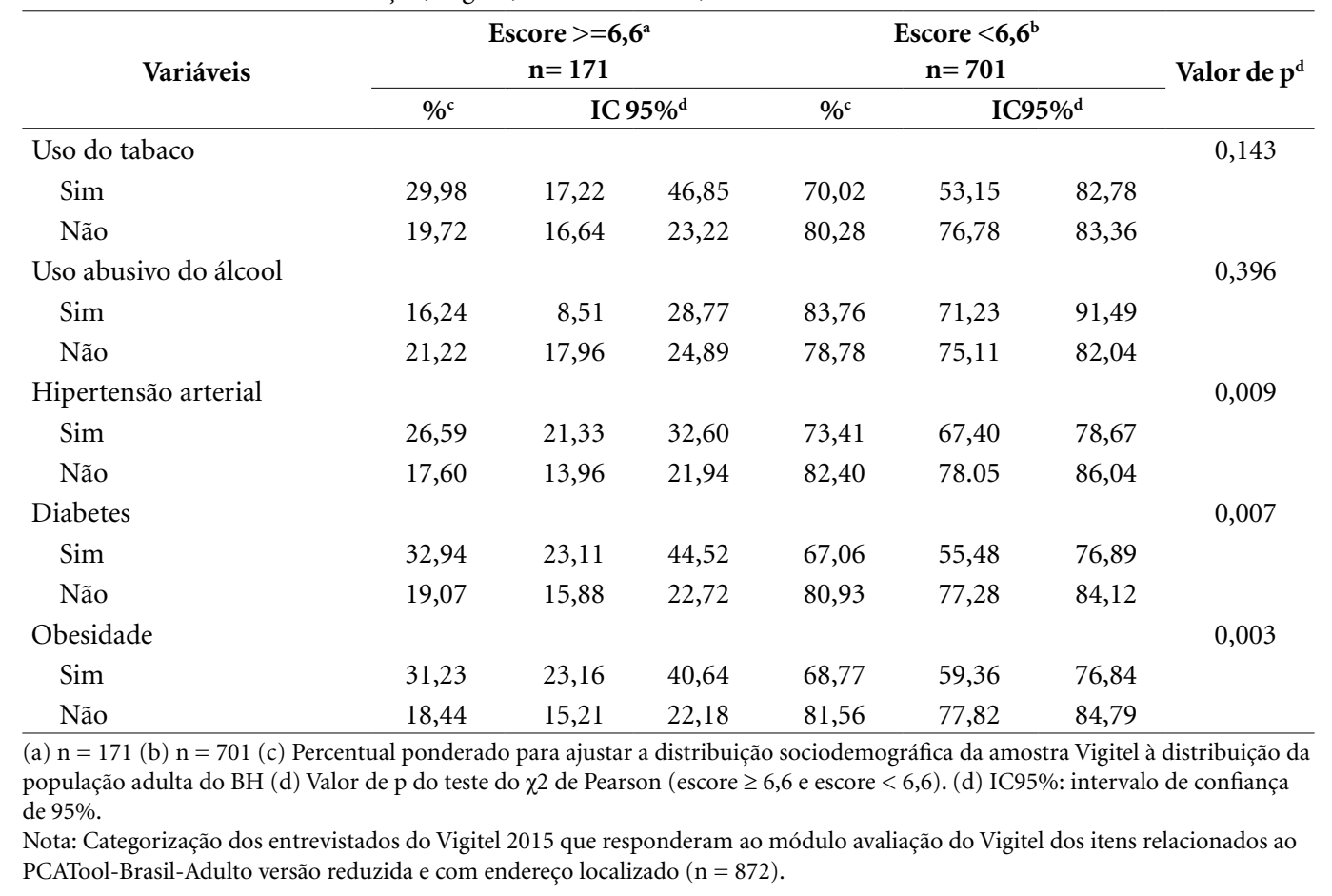

\section{Discussão}

O estudo de base populacional, a partir de entrevistas telefônicas, apresenta a avaliação do desempenho dos serviços da APS na perspectiva dos usuários em Belo Horizonte, utilizando o instrumento PCAtool-Brasil versão reduzida ${ }^{23}$.

O estudo inova ao aplicar o PCATool em uma amostra populacional em Belo Horizonte, por entrevistas telefônicas buscando avaliar o desempenho da APS e sua associação com as características sociodemográficas, condições de saúde autorreferidas e fatores de risco comportamentais para DCNT, o que difere da maioria dos estudos publicados.

O estudo permite identificar não somente o escore de avaliação dos atributos na ótica dos usuários, mas também conhecer o perfil de utilização dos serviços da APS e fatores associados ao uso $^{20,31}$. Vale ressaltar que estudos que apliquem o PCATool e analisem o escore obtido com os estilos de vida e morbidade dos usuários ainda são escassos no país.

A análise utilizando o modelo de regressão logística múltipla mostra que o escore geral é melhor avaliado pelos usuários dos serviços da APS e apresenta associação com usuários idosos (com 60 anos e mais), de baixa escolaridade, que não têm plano de saúde, e com fatores de risco comportamentais para DCNT ou doença autorreferida, como diabetes e obesidade.

Considerando o instrumento escolhido neste estudo para a avaliação de desempenho dos serviços de APS em Belo Horizonte, Prates et al..$^{32}$, em revisão sistemática em âmbito mundial, buscaram estudos publicados entre os anos de $2007 \mathrm{e}$ 2015 sobre a utilização do instrumento PCATool na perspectiva do usuário para a avaliação do desempenho da Atenção Primária à Saúde. Constataram que vários países utilizaram o PCATool, tais como, Canadá, Espanha, Coreia e China, entretanto estudos que avaliem a APS sobre a ótica dos usuários no Brasil ainda são escassos ${ }^{32,33}$.

Os resultados apontam predominância de idosos que melhor avaliaram a APS (escore $\geq$ $6,6)$. As evidências apontam que idosos apresentam mais multimorbidade e consequentemente utilizam mais os serviços de saúde, em especial a APS, para atendimento individual ou em grupo, ou mesmo para aquisição de medicamentos, 
Tabela 3. Fatores associados a avaliação com escore $\geq 6,6$ feita pelos usuários dos serviços de Atenção Primária à Saúde, Belo Horizonte. Vigitel, 2015.

\begin{tabular}{|c|c|c|c|c|c|c|c|c|}
\hline \multirow[b]{2}{*}{ Variáveis } & \multicolumn{4}{|c|}{ Modelo bruto } & \multicolumn{4}{|c|}{ Modelo ajustado } \\
\hline & $\begin{array}{c}\mathrm{OR}^{\mathrm{a}} \\
\text { (bruto) }\end{array}$ & \multicolumn{2}{|c|}{$\mathrm{IC}^{\mathrm{b}}(95 \%)$} & valor $\mathbf{p}^{\mathrm{c}}$ & $\begin{array}{c}\text { OR }^{\mathrm{d}} \\
\text { (ajustada) }\end{array}$ & \multicolumn{2}{|c|}{$\mathrm{IC}^{\mathrm{b}}(\mathbf{9 5 \%})$} & valor $\mathbf{p}^{\mathrm{c}}$ \\
\hline Masculino & 1,00 & & & & 1,00 & & & \\
\hline Feminino & 0,77 & 0,51 & 1,17 & 0,226 & 0,77 & 0,51 & 1,18 & 0,236 \\
\hline \multicolumn{9}{|l|}{ Faixa etária $(\operatorname{anos})^{\mathrm{f}}$} \\
\hline 18 a 29 & 1,00 & & & & 1,00 & & & \\
\hline 30 a 39 & 1,33 & 0,58 & 3,02 & 0,499 & 1,37 & 0,60 & 3,13 & 0,461 \\
\hline 40 a 59 & 1,19 & 0,95 & 1,50 & 0,137 & 1,20 & 0,95 & 1,52 & 0,125 \\
\hline 60 ou mais & 1,19 & 1,00 & 1,41 & 0,046 & 1,19 & 1,00 & 1,41 & 0,048 \\
\hline \multicolumn{9}{|c|}{ Escolaridade (anos de estudo) } \\
\hline 12 ou mais & 1,00 & & & & 1,00 & & & \\
\hline 9 a 11 & 1,93 & 1,01 & 3,69 & 0,045 & 2,00 & 1,03 & 3,89 & 0,042 \\
\hline 0 a 8 & 3,10 & 1,66 & 5,79 & 0,000 & 2,81 & 1,48 & 5,32 & 0,002 \\
\hline \multicolumn{9}{|l|}{$\mathrm{Raça} / \operatorname{cor}^{\mathrm{f}}$} \\
\hline Branca & 1,00 & & & & 1,00 & & & \\
\hline Preta & 1,03 & 0,55 & 1,91 & 0,936 & 1,10 & 0,58 & 2,07 & 0,775 \\
\hline Amarela & 0,55 & 0,14 & 2,18 & 0,395 & 0,66 & 0,17 & 2,59 & 0,549 \\
\hline Parda & 1,40 & 0,90 & 2,18 & 0,137 & 1,42 & 0,91 & 2,21 & 0,126 \\
\hline Indígena & 0,43 & 0,05 & 3,67 & 0,443 & 0,42 & 0,05 & 3,69 & 0,435 \\
\hline \multicolumn{9}{|l|}{ Situação Conjugal } \\
\hline Com companheiro(a) & 1,00 & & & & 1,00 & & & \\
\hline Sem companheiro(a) & 1,26 & 0,84 & 1,89 & 0,265 & 1,03 & 0,64 & 1,66 & 0,894 \\
\hline \multicolumn{9}{|l|}{ Plano de saúde } \\
\hline Sim & 1,00 & & & & 1,00 & & & \\
\hline Não & 3,26 & 2,11 & 5,03 & 0,000 & 3,21 & 2,08 & 4,96 & 0,000 \\
\hline \multicolumn{9}{|l|}{ Uso do tabaco } \\
\hline Não & 1,00 & & & & 1,00 & & & \\
\hline Sim & 1,74 & 0,82 & 3,69 & 0,147 & 1,69 & 0,80 & 3,56 & 0,169 \\
\hline \multicolumn{9}{|l|}{ Uso abusivo do álcool } \\
\hline Não & 1,00 & & & & 1,00 & & & \\
\hline Sim & 0,72 & 0,34 & 1,54 & 0,397 & 0,71 & 0,33 & 1,52 & 0,378 \\
\hline \multicolumn{9}{|l|}{ Hipertensão arterial } \\
\hline Não & 1,00 & & & & 1,00 & & & \\
\hline $\operatorname{Sim}$ & 1,70 & 1,14 & 2,53 & 0,010 & 1,49 & 0,93 & 2,40 & 0,095 \\
\hline \multicolumn{9}{|l|}{ Diabetes } \\
\hline Não & 1,00 & & & & 1,00 & & & \\
\hline Sim & 2,08 & 1,22 & 3,57 & 0,008 & 1,84 & 1,05 & 3,24 & 0,034 \\
\hline \multicolumn{9}{|l|}{ Obesidade } \\
\hline Não & 1,00 & & & & 1,00 & & & \\
\hline Sim & 2,04 & 1,28 & 3,27 & 0,003 & 1,97 & 1,20 & 3,24 & 0,007 \\
\hline
\end{tabular}

criando assim vínculo com o serviço e com as equipes, favorecendo uma melhor avaliação da assistência prestada ${ }^{17,20,35,35}$.
Pilger et al. $^{36}$, em inquérito domiciliar para analisar o padrão de utilização dos serviços de saúde por idosos nos serviços públicos no muni- 
cípio de Guarapuava no estado do Paraná, concluíram que essa população é grande usuária dos serviços de saúde.

Dotto et al. ${ }^{37}$ avaliaram a orientação dos serviços de APS e compararam a qualidade da APS entre as UBS e Unidades de Saúde da Família (USF), segundo a experiência de utilização dos idosos utilizando o PCATool, em dois distritos de Porto Alegre, Rio Grande do Sul, e identificaram que a maioria $(77,9 \%)$ utilizava os serviços de UBS e, quanto à qualidade dos serviços, observaram que $22,9 \%$ dos idosos avaliavam a APS com alto escore.

Os usuários com baixa escolaridade e sem plano de saúde também avaliam melhor os serviços da APS corroborando com outros estudos que apontam que as pessoas com baixa escolaridade e sem plano de saúde usam mais os serviços da APS, pois estas em sua maioria são dependentes do SUS $^{20}$. Os dados da PNS mostram que as coberturas de ESF são mais elevadas entre populações de baixa escolaridade. Vale ressaltar que os resultados encontrados mostram o potencial da contribuição dos serviços da APS na redução das iniquidades em saúde, promovendo maior acesso à atenção à saúde $e^{15,31,38,39}$. Entretanto, o estudo de Perillo et al. ${ }^{20}$ registra que $45,22 \%$ dos usuários com posse de plano de saúde também utilizaram os serviços da APS, o que reforça a abrangência desses serviços.

Augusto et al..$^{40} \mathrm{em}$ seu estudo apresenta que os idosos que não possuem plano privado de saúde, residentes na Região Metropolitana de Belo Horizonte, mostraram melhor avaliação nos atributos coordenação do cuidado, acesso de primeiro contato e integralidade, e pior avaliação no atributo de orientação comunitária. Constatam ainda que os idosos mais velhos, as mulheres e os de maior escolaridade avaliaram melhor o serviço. Já aqueles que reportaram maior uso do serviço e ocorrência de condições crônicas fizeram uma pior avaliação da APS. Os autores concluem que piores condições de saúde e maior uso dos serviços estão associados à percepção mais negativa dos atributos da APS entre idosos ${ }^{40}$.

Araújo et al. ${ }^{41}$ avaliam a qualidade do cuidado na APS ofertado à pessoa idosa segundo a sua perspectiva em município da região metropolitana de Natal/RN, identificam que os fatores sociodemográficos ligados à vulnerabilidade (menor renda, área rural e maior idade) associam-se positivamente a diferentes atributos da APS.

Em relação à avaliação positiva dos usuários com DCNT, tais como diabetes e obesidade, mostra que os serviços da APS têm papel fundamen- tal na vigilância das DCNT e monitoramento dos fatores de risco, pois buscam desenvolver atividades de prevenção desses agravos, promoção da saúde, além da redução de danos. Esses usuários necessitam de um monitoramento contínuo, intercorrências com especialistas, além da obtenção de insumos. A APS, assim, tem um papel importante na articulação dos pontos de atenção da RAS, garantindo os princípios da integralidade e coordenação do cuidado.

Sala et al. ${ }^{42}$, em um estudo que avalia o atributo integralidade nos serviços da APS na perspectiva dos usuários de unidades de saúde do município de São Paulo, mostra avaliação bastante favorável nas questões de porta de entrada, elenco de serviços e coordenação.

Não obstante, vale ressaltar a importância de investimentos para o fortalecimento da APS para a redução efetiva das DCNT $^{7,43}$.

Estudos apontam que a melhor avaliação dos usuários diabéticos dos serviços de APS pode estar relacionado à baixa exigência individual deles. Além disso, um sentimento de gratidão poderia impedir que os usuários avaliassem mais criticamente os serviços recebidos devido ao receio de fragilizar o vínculo com a equipe de saúde e limitar o acesso aos cuidados recebidos ou à aquisição de insumos ${ }^{44}$.

Uma limitação deste estudo se refere a um possível viés de seleção originado no uso do cadastro de linhas telefônicas fixas, que foi minimizado com o uso da ponderação e pesos pós-estratificação, ajustando a composição da amostra às características demográficas da população do município.

Sabe-se que a escolha da utilização do PCATool-adulto-Brasil versão reduzida na perspectiva do usuário apresenta limitações. A primeira seria a utilização apenas da experiência dos atores envolvidos (no caso deste estudo os usuários) na atenção como critério avaliador, não incorporando, por exemplo, a avaliação técnica do serviço prestado. Entretanto, considerando que a opinião dos usuários dos serviços da APS é importante no processo avaliativo do serviço e que o inquérito telefônico pode ser útil no levantamento dos dados e é um processo de baixo custo pode-se realizar outros estudos com os profissionais de forma a complementar a avaliação. A segunda aponta uma limitação no que se refere ao fato deste instrumento não ter sido desenvolvido para a análise de escores por atributo, sendo capaz de medir a presença e a extensão dos atributos essenciais e derivados da APS por meio do escore geral. Entretanto, deve-se levar em consideração 
a viabilidade de utilização da versão completa ou mesmo da realização de adaptações na versão reduzida para uso em inquéritos telefônicos.

A avaliação torna-se um importante instrumento para a tomada de decisão pelos profissionais, gestores e acadêmicos ${ }^{45-48}$, devendo ser incorporada na gestão, especialmente local.

Este estudo apresenta uma nova possibilidade de uso da avaliação dos serviços de saúde, em especial da APS, por meio do inquérito telefônico, que pode ser uma boa estratégia de monitoramento, captando o olhar do usuário, com menor custo e rapidez. Além disto, o instrumento PCATool - Brasil é uma importante ferramenta na avaliação da qualidade dos serviços na APS, considerando os aspectos estruturais e de processos nos serviços de saúde e possibilita estabelecer associações a desfechos clínicos.

\section{Conclusão}

O estudo inova ao utilizar o PCATool em inquérito telefônico e mostrou-se útil na avaliação do desempenho da APS no município de Belo Horizonte, na perspectiva dos usuários e a sua associação com as características sociodemográficas, condições autorreferidas e fatores de risco comportamentais para DCNT.

Vale ressaltar a necessidade de realização de mais estudos que avaliem o desempenho da APS na perspectiva do usuário, bem como estudos que apresentem análise relacionando a avaliação do desempenho da APS com os desfechos clínicos.

$O$ estudo mostra ser uma forma inovadora de avaliação e que pode ser replicada em todo país, além de contribuir na gestão dos serviços, além de ser de baixo custo e rapidez na aplicação do instrumento. Como é uma ferramenta utilizada mundialmente com diferentes versões validadas para contextos locais, permite a comparabilidade dos achados.

\section{Colaboradores}

RD Perillo e DC Malta trabalharam na concepção do estudo, na redação do artigo, na análise e interpretação dos dados, na revisão de literatura e na revisão final do texto. RTI Bernal e KC Poças participaram da concepção do estudo, da análise e interpretação dos dados e da revisão final do texto. EC Duarte participou da concepção do estudo e da revisão final do texto. Todos os autores aprovaram sua versão final.

\section{Agradecimentos}

Os autores agradecem ao Ministério da Saúde, Secretaria de Vigilância em Saúde, pelo financiamento por meio de TED. Malta DC agradece ao CNPq pela bolsa de produtividade em pesquisa. 


\section{Referências}

1. Macinko J, Mendonça CS. Estratégia Saúde da Família, um forte modelo de atenção primária à saúde que traz resultados. Saúde debate 2018; 42(1):18-37.

2. Macinko J, Oliveira VB, Turci MA, Guanais FC, Bonolo PF, Lima-Costa MF. The influence of primary care and hospital supply on ambulatory care-sensitive hospitalizations among adults in Brazil, 1999-2007. Am J Public Health [Internet]. 2011 Oct [acessado 2020 Ago 4]; 101(10):1963-1970. Disponível em: https://www.ncbi.nlm.nih.gov/pubmed/21330584

3. Starfield B, Shi L. Manual for the Primary Care Assessment Tools. Baltimore: Johns Hopkins University; 2002.

4. Starfield B. Atenção primária - Equilíbrio entre necessidades de saúde, serviços e tecnologia. $2^{\mathrm{a}}$ ed. Brasília: Organização das Nações Unidas para a Educação, a Ciência e a Cultura, Ministério da Saúde do Brasil; 2004.

5. Tasca R, Massuda A, Carvalho WM, Buchweitz C, Harzheim E. Recomendações para o fortalecimento da atenção primária à saúde no Brasil. Rev Panam Salud Publica 2020; 44:e4.

6. Campos RTO, Ferrer AL, Gama CAP, Campos GWS, Trapé TL, Dantas DV. Avaliação da qualidade do acesso na atenção primária de uma grande cidade brasileira na perspectiva dos usuários. Saúde debate [Internet]. 2014 Oct [acessado 2020 Ago 4]; 38(n. esp.):252-264. Disponível em: http:// www.scielo.br/scielo.php?script=sci_arttext\&pi$\mathrm{d}=$ S0103-11042014000600252\&lng $=$ en

7. Gontijo TL, Duarte AGS, Guimarães EAA, Silva J. Avaliação da atenção primária: o ponto de vista de usuários. Saúde Debate 2017; 41(114):741-752.

8. Garcia LAA, Nardelli GG, Oliveira AFM, Casaburi LE, Camargo FC, Santos AS. Satisfação de idosos octogenários com os serviços de Atenção Primária à Saúde. Rev. bras. geriatr. gerontol. [Internet]. 2020 [cited 2020 Oct 26]; 23(1):e190235. Disponível em: http:// www.scielo.br/scielo.php?script $=$ sci_arttext\&pi$\mathrm{d}=$ S1809-98232020000100204\&lng=en

9. Reis JG, Harzheim E, Nachif MCA, Freitas JC, D’Ávila O, Hauser L, Martins C, Pedebos LA, Pinto LF. Criação da Secretaria de Atenção Primária à Saúde e suas implicações para o SUS. Cien Saude Colet [Internet]. 2019 Sep [cited 2020 Oct 27]; 24(9):3457-3462. Disponível em: http://www.scielo.br/scielo.php?script=sci_arttext\&pid=S1413-81232019000903457\&lng=en

10. Fracolli LA, Gomes MFP, Nabão FRZ, Santos MS, Capellini, VK, Almeida ACC. Instrumentos de avaliação da atenção primária à saúde: Revisão de literatura e metassíntese. Cien Saude Colet 2014; 19(12):48514860.

11. Brasil. Ministério da Saúde (MS). Manual do instrumento de avaliação da atenção primária à saúde: Primary Care Assessment Tool PCATool. Brasília: MS; 2010.

12. Ribeiro LA, Scatena JH. A avaliação da atenção primária à saúde no contexto brasileiro: uma análise da produção científica entre 2007 e 2017. Saude Soc 2019; 28(2):95-110.
13. Malta DC, Bernal RTI, Lima MG, Araújo SSC, Silva MMA, Freitas MIF, Barros MBA. Doenças crônicas não transmissíveis e a utilização de serviços de saúde: análise da Pesquisa Nacional de Saúde no Brasil. Rev Saude Publica [Internet]. 2017 [acessado 2020 Ago 4]; 51(Supl. 1):4s. Disponível em: http://dx.doi. org/10.1590/s1518-8787.2017051000090

14. Travassos C, Martins M. Uma revisão sobre os conceitos de acesso e utilização de serviços de saúde. Cad Saude Publica [Internet]. 2004 [acessado 2020 Ago 4]; 20(Supl. 2):S190-S198. Disponível em: http:// www.scielo.br/scielo.php?script=sci_arttext\&pi$\mathrm{d}=$ S0102-311X2004000800014\&lng=en

15. Stopa SR, Malta DC, Monteiro, CN, Szwarcwald CL, Goldbaum M, Cesar CLG. Acesso e uso de serviços de saúde pela população brasileira, Pesquisa Nacional de Saúde 2013. Rev Saude Publica [online] 2017; 51(Supl. 1):3s. [acessado 2020 Ago 4]. Disponível em: https:// doi.org/10.1590/S1518-8787.2017051000074

16. Malta DC, Andrade SSCA, Oliveira TP, Moura L, Prado RR, Souza MFM. Probabilidade de morte prematura por doenças crônicas não transmissíveis, Brasil e regiões, projeções para 2025. Rev. bras. epidemiol. [Internet]. 2019 [acessado 2020 Ago 4]; 22(Epub):e190030. Disponível em: http:// www.scielo.br/scielo.php?script=sci_arttext\&pi$\mathrm{d}=$ S1415-790X2019000100428\&lng=en

17. Malta DC, Santos MAS, Stopa SR, Vieira JEB, Melo AE, Reis AAC. A Cobertura da Estratégia de Saúde da Família (ESF) no Brasil, segundo a Pesquisa Nacional de Saúde, 2013. Cien Saude Colet [online]. 2016; 21(2):327-338. [acessado 2020 Ago 4]. Disponível em: https://doi.org/10.1590/1413-81232015212.23602015

18. Brasil. Ministério da Saúde (MS). Vigitel Brasil 2015: vigilância de fatores de risco e proteção para doenças crônicas por inquérito telefônico: estimativas sobre frequência e distribuição sociodemográfica de fatores de risco e proteção para doenças crônicas nas capitais dos 26 estados brasileiros e no Distrito Federal em 2015. Brasília: MS; 2016.

19. Belo Horizonte. Secretaria Municipal de Saúde. Relatório Anual de Gestão 2019. 2020. 136p. Disponível em: https://prefeitura.pbh.gov.br/controladoria/relatorios-de-gestao-2019.

20. Perillo RD, Poças KC, Machado IE, Bernal RTI, Duarte EC, Malta DC. Fatores associados à utilização da atenção primária pela população adulta de Belo Horizonte, Minas Gerais, segundo inquérito telefônico. REME - Rev Min Enferm. 2020 [acessado 2020 Ago 4]; 24:e-1300. Disponível em: https://www.reme.org.br/ artigo/detalhes/1446

21. Poças KC. Avaliação da Atenção Primária À Saúde no Distrito Federal [tese]. Brasília: Universidade de Brasília; 2017.

22. Perillo RD, Bernal RTI, Poças KC, Duarte EC, Malta DC. Avaliação da Atenção Primária à Saúde na ótica dos usuários: reflexões sobre o uso do Primary Care Assessment Tool-Brasil versão reduzida nos inquéritos telefônicos. Rev. bras. epidemiol. 2020; 23(Supl. 1):e200013. [acessado 2020 Ago 4]. Disponível em: https://doi.org/10.1590/1980-549720200013 
23. Oliveira MMC, Harzheim E, Riboldi, J, Duncan BB. PCATool-ADULTO-BRASIL: uma versão reduzida. Rev Bras Med Fam Comunidade 2013 [acessado 2020 Ago 4]; 8(29):256-263. Disponível em: https://www. rbmfc.org.br/rbmfc/article/view/823

24. Shi L, Starfield B, Jiahong, X. Validating the Adult Primary Care Assessment Tool. The Journal of Family Practice 2001; 50(2):161-175.

25. Donabedian A. Evaluacion de la calidad de la atención médica. Revista de Calidad Asistencial 2001; 16:S11-S27.

26. Almeida C, Macinko J. Validação de uma metodologia de avaliação rápida das características organizacionais $e$ do desempenho dos serviços de atenção básica do Sistema Único de Saúde (SUS) em nível local. Brasília: OPAS, OMS, Ministério da Saúde do Brasil; 2006. (Série Técnica - Desenvolvimento de Sistemas e Serviços de Saúde no 10).

27. Harzheim E, Gonçalves MR, D’Avila OP, Hauser L, Pinto LF. Estudos do PCATool no Brasil. In: Mendonça MHM, Matta GC, Gondim R, Giovanella L, organizadores. Atenção primária à saúde no Brasil: conceitos, práticas e pesquisa. Rio de Janeiro: SciELO-Editora Fiocruz; 2018. p. 493-526.

28. Bernal RTI, Iser BPM, Malta DC, Claro RM. Sistema de Vigilância de Fatores de Risco e Proteção para Doenças Crônicas por Inquérito Telefônico (Vigitel): mudança na metodologia de ponderação. Epidemiol Serv Saúde 2017 [acessado 2020 Ago 4]; 26(4):70112. Disponível em: http://www.scielo.br/pdf/ress/ v26n4/2237-9622-ress-26-04-00701.pdf

29. Flores-Cervantes I, Brick JM, Jones ME. Weighting for nontelephone household in the 2001 California Health Interview Survey. Joint Statistical Meetings - Section on Survey Research Methods, 2002. p. 1002-1007.

30. Nick W. SURVWGT: Stata module to create and manipulate survey weights. In: Statistical Software Components. Revised 11 Feb 2018. Boston: Boston College Department of Economics; 2002.

31. Poças KC, Perillo RD, Bernal RTI, Malta DC, Duarte EC. Primeira escolha para utilização de serviços de saúde pela população adulta do Distrito Federal, 2015: um inquérito de base populacional. Epidemiol. Serv. Saúde [Internet]. 2019 Jun [acessado 2020 Ago 4]; 28(2):e2018124. Disponível em: http://scielo.iec.gov.br/scielo.php?script=sci_arttext\&pid=S1679-49742019000200024\&lng=pt. Epub 29-Jul-2019. http://dx.doi.org/10.5123/s167949742019000200017.

32. Prates ML, Machado JC, Silva LS, Avelar OS, Prates LL, Mendonça ET, Costa GD, Cotta RMM. Desempenho da Atenção Primária à Saúde segundo o instrumento PCATool: uma revisão sistemática. Cien Saude Colet 2017; 22(6):1881-1893.

33. Wkas P, Samico IC, Caminha MFC, Batista Filho M, Silva SL. Primary health care assessment from the users' perspectives: a systematic review. Rev Esc Enferm USP 2016; 50(2):331-340.
34. Nunes BP, Batista SRR, Andrade FB, Souza Junior PRB, Lima-Costa MF, Facchini LA. Multimorbidade em indivíduos com 50 anos ou mais de idade: ELSI-Brasil. Rev Saude Publica [online]. 2018; 52(Supl. 2):10s. [acessado 2020 Ago 4]. Disponível em: https:// doi.org/10.11606/S1518-8787.2018052000637

35. Melo LA, Lima KC. Prevalência e fatores associados a multimorbidades em idosos brasileiros. Cien Saude Colet 2018; 25(10):3869-3877.

36. Pilger C, Menon MU, Mathias TAF. Utilização de serviços de saúde por idosos vivendo na comunidade. Rev. esc. enferm. USP [Internet]. $2013 \mathrm{Feb}$ [acessado 2020 Ago 4]; 47(1):213-220. Disponível em: http:// www.scielo.br/scielo.php?script=sci_arttext\&pi$\mathrm{d}=$ S0080-62342013000100027\&lng=en

37. Dotto J, Ávila G, Martins A, Hugo F, D’Avila O, Hilgert J. Avaliação da qualidade dos serviços de atenção primária à saúde acessados por idosos em dois distritos de Porto Alegre, RS, Brasil. Revista Da Faculdade De Odontologia - UPF 2016; 21(1). [acessado 2020 Ago 4]. Disponível em: https://doi.org/10.5335/rfo. v21i1.5385

38. Turci MA, Lima-Costa MF, Macinko J. Influência de fatores estruturais e organizacionais no desempenho da atenção primária à saúde em Belo Horizonte, Minas Gerais, Brasil, na avaliação de gestores e enfermeiros. Cad Saude Publica [Internet]. 2015 Set [acessado 2020 Ago 4]; 31(9):1941-1952. Disponível em: http:// www.scielo.br/scielo.php?script=sci_arttext\&pi$\mathrm{d}=$ S0102-311X2015000901941\&lng=en

39. Malta DC, Stopa SR, Pinto B, Iser M, Bernal RTI, Claro RM, Nardi ACF, Reis AAC, Monteiro CA. Fatores de risco e proteção para doenças crônicas por inquérito telefônico nas capitais brasileiras, Vigitel 2014. Rev. bras. epidemiol. 2015; 18(Supl. 2):238-255.

40. Augusto DK, Lima-Costa MF, Macinko J, Peixoto SV. Fatores associados à avaliação da qualidade da atenção primária à saúde por idosos residentes na Região Metropolitana de Belo Horizonte, Minas Gerais, 2010. Epidemiol. Serv. Saúde [Internet]. 2019 [acessado 2020 Ago 4]; 28(1):e2018128. Disponível em: http:// www.scielo.br/scielo.php?script=sci_arttext\&pi$\mathrm{d}=$ S2237-96222019000100316\&lng $=$ en

41. Araújo LUA, Gama ZAS, Nascimento FLA, Oliveira HFV, Azevedo WM, Almeida Júnior HJB. Avaliação da qualidade da atenção primária à saúde sob a perspectiva do idoso. Cien Saude Colet [Internet]. 2014 Aug [acessado 2020 Ago 17]; 19(8):3521-3532. Disponível em: http://www.scielo.br/scielo.php?script=sci_arttext\&pid=S1413-81232014000803521\&lng=en

42. Sala A, Luppi CG, Simões O, Marsiglia RG. Integralidade e Atenção Primária à Saúde: avaliação na perspectiva dos usuários de unidades de saúde do município de São Paulo. Saude soc 2011; 20(4):948960. Disponível em: https://www.scielosp.org/article/ sausoc/2011.v20n4/948-960/pt/

43. Almeida LM, Ramos KFC, Randow R, Guerra VA. Estratégias e desafios da gestão da Atenção Primária à Saúde no controle e prevenção da obesidade. Rev. GઐS [Internet]. [acessado 2020 Ago 4]; 8(1):114-139. Disponível em: https://periodicos.unb.br/index.php/ rgs/article/view/3700 
44. Saes M, Facchini L, Tomasi E. Avaliação da satisfação de usuários da Atenção Básica portadores de hipertensão e diabetes. APS Em Revista 2019; 1(3):206-221.

45. Tanaka OY, Tamaki EM. O papel da avaliação para a tomada de decisão na gestão de serviços de saúde. CIen Saude Colet [Internet]. 2012 Abr [acessado 2020 Ago 4]; 17(4):821-828. Disponível em: http:// www.scielo.br/scielo.php?script $=$ sci_arttext\&pi$\mathrm{d}=$ S1413-81232012000400002\&lng=en

46. Macinko J, Guanais FC, Souza MFM. Evaluation of the impact of the Family Health Program on infant mortality in Brazil, 1990-2002. J Epidemiol Community Health 2006; 60(1):13-20.

47. Stein AT. A avaliação dos serviços de saúde deve ser realizada com instrumentos validados. Epidemiol. Serv. Saúde [Internet]. 2013 Mar [acessado 2020 Ago 4]; 22(1):179-181. Disponível em: http:// scielo.iec.gov.br/scielo.php?script=sci_arttext\&pi$\mathrm{d}=$ S1679-49742013000100019\&lng $=\mathrm{pt}$

48. Pinto Junior EP, Cavalcante JLM, Sousa RA, Morais APP, Silva MGC. Análise da produção científica sobre avaliação, no contexto da saúde da família, em periódicos brasileiros. Saúde debate [Internet]. $2015 \mathrm{Mar}$ [acessado 2020 Ago 4]; 39(104):268-278. Disponível em: http://www.scielo.br/scielo.php?script=sci_arttext\&pid=S0103-11042015000100268\&lng=en

Artigo apresentado em 11/12/2020

Aprovado em 11/12/2020

Versão final apresentada em 13/12/2020

Editores-chefes: Maria Cecília de Souza Minayo, Romeu Gomes, Antônio Augusto Moura da Silva

\section{Errata}

p. 961

\section{Onde se lê}

\section{ARTIGO ARTICLE}

\section{Leia-se}

TEMAS LIVRES FREE THEMES

Revista Ciência \& Saúde Coletiva 2021; 26(5): 1991-1991. 